\title{
Studies on Malarial Sporozoites. II. Effect of Age and Dosage of Sporozoites on Their Infectiousness
}

\section{Richard J. Porter, Raymond L. Laird and Elizabeth M. Dusseau}

Department of Tropical Diseases, School of Public Health, University of Michigan, Ann Arbor, Michigan

(Submitted for publication, 10 July 1953)

EPITOME

The significance of dosage of sporozoites in malarial infections has not been accessible to accurate quantitative study. The inoculation methods of Porter, Laird and Dusseau, however, furnished a tool for investigation of the problem. Exploitation of this opportunity has shown a rather consistent relationship between the numbers of sporozoites inoculated and the intensity of resulting infections. Further, and more important, it has revealed a progrcssive decrease in numbers and infectiousness of sporozoites during their sojourn in mosquitoes.

Sporozoites of Plasmodium gallinaceum were obtained from Aedes aegypti or Aedes albopictus mosquitoes kept at about $28^{\circ} \mathrm{C}\left(27-30^{\circ}\right)$ for various periods after infection. Chickens were inoculated intravenously with known numbers of these sporozoites and the intensities of the ensuing infections were compared on the basis of the proportion of parasitized erythrocytes six days after inoculation. Experiments with serial dilution showed that the parasitemias on the sixth day were proportional to the dosage of sporozoites. Therefore, all data were converted to a single value, the number of parasitized erythrocytes (per 10,000) on the sixth day, per million sporozoites inoculated. The relative constancy of this ratio for a given preparation, regardless of the actual dosage of sporozoites, makes it suitable for comparison between different preparations.

Figure 1 shows the relationships between duration of infection in mosquitoes and (S/M) number of sporozoites recovered per mosquito dissected, $(\mathrm{P} / \mathrm{S})$ parasitemia per million sporozoites inoculated and $(\mathrm{P} / \mathrm{M})$ parasitemia per mosquito inoculated. All curves are based on weekly logarithmic averages. A total of 109 lots of mosquitoes is included. Three 


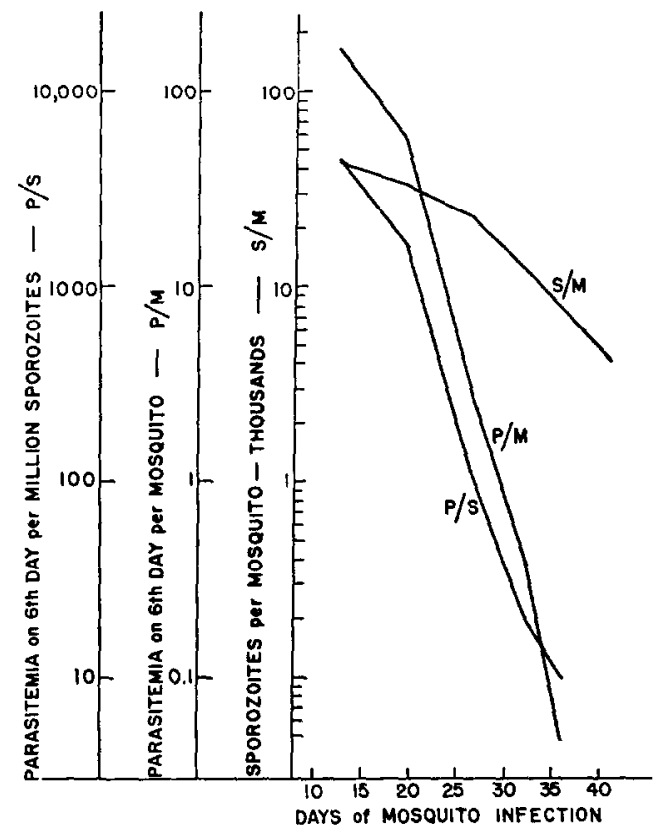

Frg. 1. Numbers and infectivity of sporozoites in relation to duration of infection in mosquitoes. Logarithmic means.

facts, also supported by the additional data recorded in the Amplification (see below), are apparent from the graph. The number of sporozoites recovered per mosquito fell about $90 \%$ in 4 weeks. The intensity of infection in chicks per million sporozoites inoculated declined at a rate of about $90 \%$ per week until, after 4 weeks, very large doses of sporozoites often failed to produce infection. Finally, the intensity of infection per mosquito inoculated decreased during 4 weeks to about one ten-thousandth its original value.

Samples of mosquitoes tested during the first week ( 9 to 15 days after infection) showed a mean value of 4200 parasitized erythrocytes (per $10,000)$ per million sporozoites inoculated. The extreme range was $700-$ 20,000 , and 30 of the 36 samples gave values between 2000 and 10,000 . This must represent the optimum infectivity of sporozoites of Plasmodium gallinaceum with the technique used. Since a calculated dose of 50 sporozoites did not always cause infection, many sporozoites must be non-infective under these conditions. The relative consistency of the data, however, suggests that the proportion of non-viable sporozoites is fairly constant. 
Previous work of Boyd and others has produced roughly quantitative evidence of a decline in infectivity of sporozoites during their stay in mosquitoes. The present data show for Plasmodium gallinaceum that such a decline clearly exists and that it is a result of loss in both numbers and infectivity of sporozoites.

\section{AMPLIFICATION}

\section{Materials and Methods}

The procedures for infection of chicks with sporozoites have been discussed extensively elsewhere (Porter, et al., 1952). Aedes aegypti and, to a small extent, A. albopictus were reared by the general methods of Shannon and Putnam (1934). They were infected within a week of emergence by feeding on thicks harboring a blood-passage line of the 8-A strain of Plasmodium gallinaceum. Infected mosquitoes were kept at $27-30^{\circ} \mathrm{C}$ (mean $28^{\circ}$ ) with cooked raisins or slices of apple as a maintenance diet.

For dissection, mosquitoes were immobilized with minimal exposure to anesthetic ether. The heads were cut off, and a bit of thoracic contents was squeezed out into $0.85 \%$ sodium chloride solution. The tissue thus removed usually contains the salivary glands. Glands from each lot of mosquitoes were suspended in 0.50 $\mathrm{ml}$ of heparinized normal chick blood $(0.85 \%$ saline solution, $0.05 \mathrm{cc}$; powdered heparin, about $20 \mathrm{mg}$; blood, $0.45 \mathrm{ml}$ ) in a glass roller mill rotated for 30 minutes.

Dried, Giemsa-stained films of the preparation were examined to determine the ratio of sporozoites to erythrocytes. At least 10 sporozoites were counted unless a total of 10,000 erythrocytes was reached first. This gives a figure ( sporozoites per 10,000 erythrocytes) with a probable error of $20 \%$ or less for values of 10 or more. For smaller values, the probable error is progressively larger. The total number of sporozoites in the suspension can be calculated from the sufficiently accurate assumption that $0.45 \mathrm{ml}$ of normal chick blood contains about $1,000,000,000$ erythrocytes. Each suspension contained the salivary glands from 9 to 177 , usually $10-20$ mosquitoes. Aliquots of the preparation were inoculated intravenously to White Rock or White Leghorn chicks, 2-7 days old, in groups of four or more.

Parasitemias were determined from daily blood films of inoculated chicks, the values obtained on the sixth day after inoculation being used for comparison between different groups. When these values were too low they were calculated from the parasitemias on the seventh day, which, in these experiments, were about 10 times those for the sixth day.

\section{SUPPLEMENTARY Results}

Figure 2 shows the numbers of sporozoites recovered per mosquito in 109 samples of $A$. aegypti and 8 of $A$. albopictus, plotted against the duration of infection in mosquitoes. The considerable variation is probably a result of both variation in initial level of infection and irregular recovery of sporozoites by the suspending method. It does not obscure the trend, which clearly reveals a progressive decline in numbers of sporo- 
zoites with increasing duration of infection. This trend is particularly obvious in the curve of logarithmic means, calculated weekly beginning with the ninth day (the last value represents all samples from the 37 th through the 48th day).

Eighteen lots of mosquitoes were tested both before the 18th and after the 28th day. Fifteen showed a reduction in number of sporozoites per mosquito between the earlier and later tests. The mean reduction for all mosquito lots was $50 \%$, with a standard error of $12.1 \%$.

Table I shows two experiments in serial dilution of suspensions of sporozoites. In both cases the mosquitoes were dissected 13 days after infection. In experiment $A$ the original suspension contained 35 million sporozoites from 177 mosquitoes. Dilutions were made in saline solution containing about $1 \%$ blood. Each group contained six chicks. In experiment $B$ the preparation contained four million sporozoites and dilutions

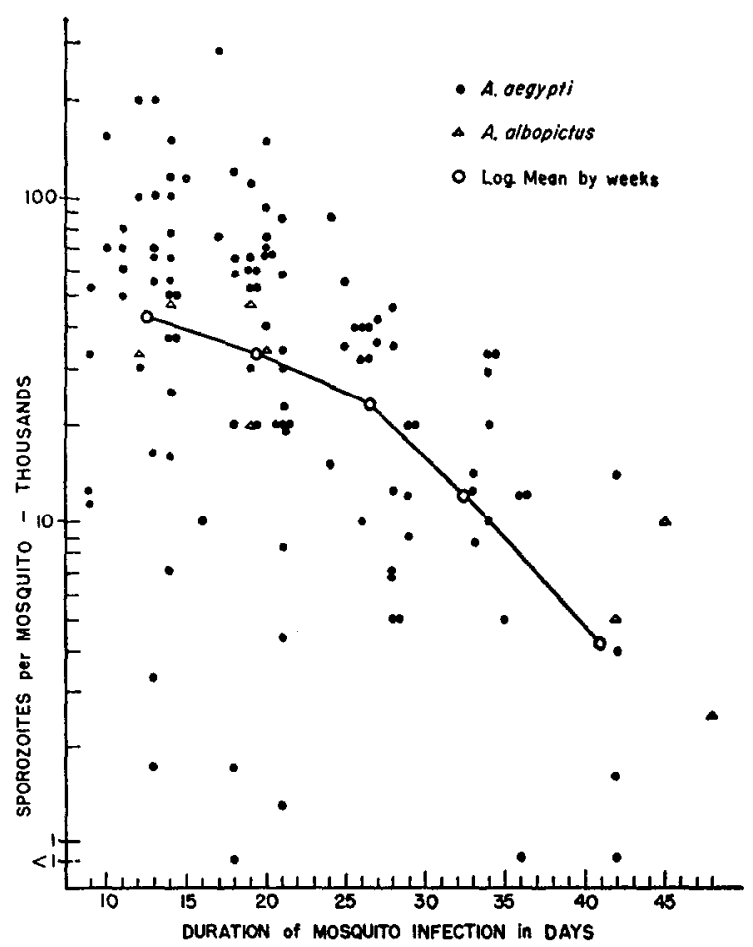

FIG. 2. Numbers of sporozoites in relation to duration of infection in mosquitoes. 
TABLE I

\begin{tabular}{ccccc}
\hline & \multicolumn{2}{c}{$\begin{array}{c}\text { Experiment A } \\
\text { Mean } \\
\text { parasitemia on } \\
\text { 6th day }\end{array}$} & $\begin{array}{c}\text { Parasitemia } \\
\text { per million } \\
\text { sporozoites }\end{array}$ & \multicolumn{2}{c}{$\begin{array}{c}\text { Experiment B } \\
\text { parasitemia } \\
\text { on 6th day }\end{array}$} & $\begin{array}{c}\text { Parasitemia } \\
\text { per million } \\
\text { sporozoites }\end{array}$ \\
\hline Sporozoites inoculated & 1275 & 2550 & 820 & 1640 \\
500,000 & 177 & 3540 & 136 & 2720 \\
50,000 & & & 35 & 3110 \\
11,250 & 14 & 2800 & & \\
5,000 & $2.2^{*}$ & 4400 & $1.7^{*}$ & 3400 \\
500 & & & & \\
\hline
\end{tabular}

- Calculated from parasitemia on the 7th day, see Materials and Methods section.

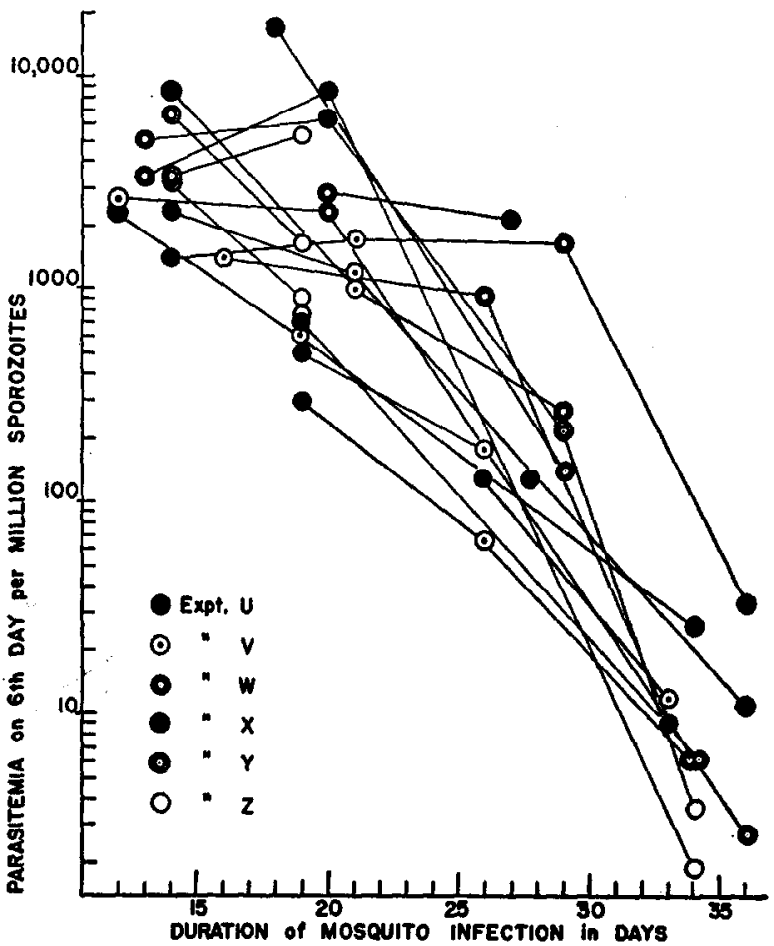

FIG. 3. Infectivity of sporozoites in relation to duration of infection in mosquitoes. Values for the same lot of mosquitoes tested in different experiments are connected by solid lines. 
were made with saline solution. The first group contained three chicks, the others, eight to ten each.

In experiment A six chicks were inoculated with 50 sporozoites each; three became infected. With doses of 5 and 0.5 sporozoites each, none became infected. The data from these two experiments show that the figure obtained by dividing the 6th day parasitemia by the number of sporozoites inoculated is relatively constant for a rather wide range of actual sporozoite dosages.

Figure 3 presents data from six experiments on infectivity of sporozoites in relation to the time elapsed after the infecting feed. Each point on the graph represents a value for a group of four or more chicks inoculated with sporozoites from nine or more mosquitoes. The values (number of parasitized cells per 10,000 erythrocytes on the sixth day, per million

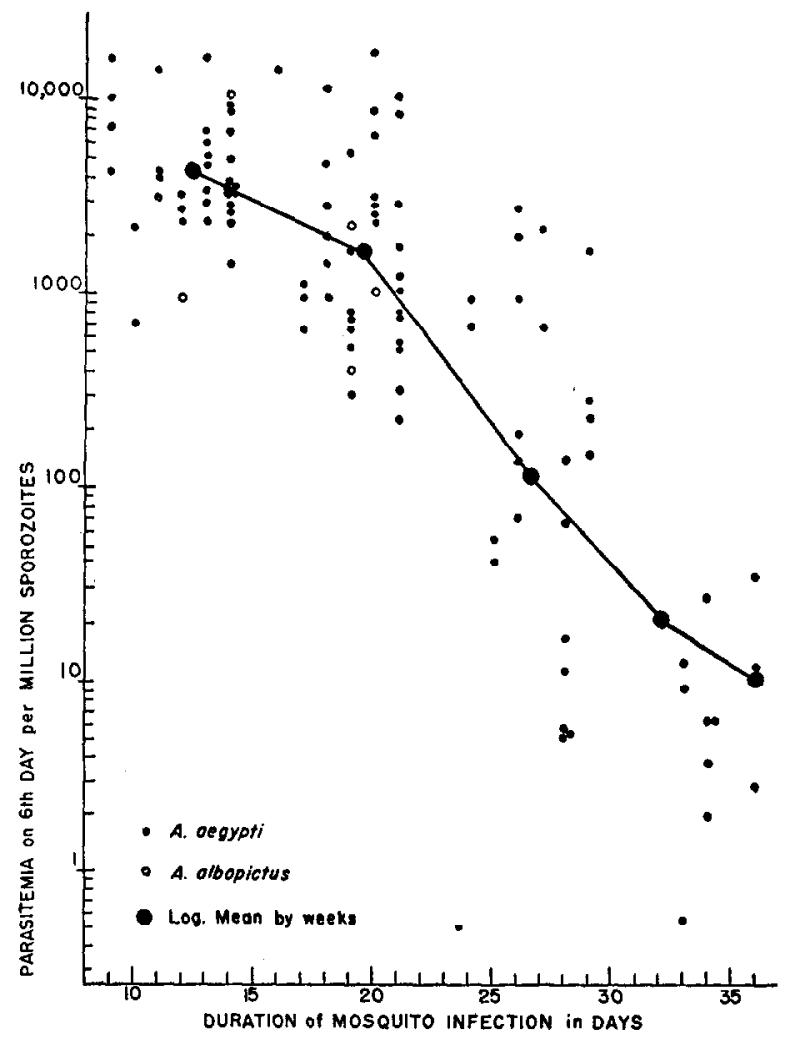

Fra. 4. Infectivity of sporozoites in relation to duration of infection in mosquitoes. 
sporozoites inoculated) for each experiment are represented by a distinctive symbol, so that infectivities of different lots of mosquitoes in one experiment may be compared. Lines connect the values for samples of each lot tested in different experiments, so that the change in infectivity of one lot from experiment to experiment may be observed. It is elcar that within each experiment the relative infectivity of sporozoites decreases with age of the infection in mosquitoes. Similarly, the infectivity of samples from the same lot, as determined in serial experiments, falls progressively with increase in duration of the infection.

Figure 4 gives the same data for all 109 mosquito samples tested, including those shown in Fig. 3. The logarithmic means show a progressive decrease in infectivity at a rate of about $90 \%$ a week. By the fifth week from the infecting feed the infectivity of sporozoites was so slight as to be nearly undetectable. In fact, in addition to the data shown in Fig. 4, there were two lots tested at 35 days and six tested at 44-48 days which produced no infection in inoculated chicks.

The data presented apply principally to Aedes aegypti. Those on $A$. albopictus are insufficient in numbers to justify conclusions other than that they show no discrepancies from those for $A$. aegypti.

\section{Discussion}

The data presented confirm the over-all loss in infectivity reported by others for human malarias. While mosquitoes have transmitted $P$. vivax up to ten weeks after infection (James, 1926) and $P$. falciparum to 55 days (Mayne, 1919, according to Boyd, 1949), there are reports of initially infective mosquito lots which became negative in as short a time as 23 days with $P$. vivax (Mitzmain, 1916) and 40 days with $P$. falciparum (Boyd et al., 1936). In some of these studies it appeared that the loss of infectivity resulted from depletion of sporozoites by repeated fecding of the mosquitoes, but in other instances (Barber, 1936; Boyd et al., 1936) microscopic studies indicated degeneration of sporozoites in the salivary glands.

The types of quantitative methods used in the present investigation have not been applied to primate malarias and are probably not appropriate. Rough measures of the viability of the sporozoites have, however, been utilized. Mitzmain (1916) found an increase in length of prepatent periods with successive applications of $A$. punctipennis harboring $P$. vivax. Boyd and Stratman-Thomas (1934) with P. vivax and Boyd et al. (1936) with $P$. falciparum reported a progressive decrease in percentage of infections in exposed paretics with increase in duration of infection in 
A. quadrimaculatus kept at $4-7^{\circ} \mathrm{C}$. No takes were obtained with $P$. vivax after 50 days nor with $P$. falciparum after 40 days from the completion of sporogony (total time from infecting feed not given).

The principal difference between the present work and that reported by others is that, by inoculation of known numbers of sporozoites, we have been able to distinguish between loss in numbers and loss of infectivity of sporozoites and to show that, in $P$. gallinaceum, at least, both occur. Since our mosquitoes received no blood meals after the infecting feed, it is clear that repeated feeding on animals was not responsible for the decline in infectivity. It should be pointed out that our data do not distinguish between effects of age of infection and those of age of the host mosquitoes. However, with P. vivax James (1926 and 1927) reinfected mosquitoes which had lost their infectivity. Since there are no major discrepancies in any of the work discussed it may be supposed that the general effects of duration of mosquito infection are similar for primate and avian malarias. We may conclude tentatively for all malarias that sporozoites decrease in numbers and infectivity during their stay in mosquitoes and that these decreases are not primarily a result of age of the mosquitocs nor of depletion of sporozoites by repeatcd blood meals.

\section{ReFerences}

BАнвен, M. A. 1936. Degeneration of sporozoites of the malaria parasite in anopheline mosquitoes in nature and its relation to the transmission of malaria. Am. J. Hyg. 24, 45-56.

Boyd, M. F. Ed. 1949. Malariology. Saunders, Philadelphia, Vol. 1, p. 640.

Boyd, M. F., and Stratman-Thomas, W. K. 1934. On the duration of infectiousness in anophelines harboring $P$. vivax. Am. J. Hyg. 19, 539 40.

Boyd, M. F., Stratman-Thomas, W. K., and Kicthen, S. F. 1936. On the duration of infectiousness in anophelines harboring P. falciparum. Am. J. Trop. Med. 16, $157-58$.

JAMEs, S. P. 1926. Epidemiological results of a laboratory study of malaria in England. Trans. Roy. Soc. Trop. Med. Hyg. 20, 143-57.

JAMEs, S. P. 1927. History of a group of Anopheles mosquitoes infected with Plasmodium vivax. Abhandl. Gebiete Auslandskunde, Hamburgische, Universität, 26 Reiche D., Medizin Bd. 2.

Mitzmain, M. B. 1916. Anopheles infectivity experiments. An attempt to determine the number of persons one mosquito can infect with malaria. Public Health Repts. 31, 2325.

Porter, R. J., Laind, R. I., ANd Dusseat, E. M. 1952. Studies on malarial sporozoites. I. Effect of various environmental conditions. Exptl. Parasitol. 1, $229-244$.

Shannon, R. C., ano Putnam, P. 1934. The biology of Stegomyia under laboratory conditions. Proc. Entomol, Soc. Washington 36, 185-242. 